\title{
Retroperitoneoscopic pyelolithotomy: A minimally invasive alternative for the management of large renal pelvic stone
}

\author{
Saurabh Sudhir Chipde, Santosh Agrawal \\ Department of Urology and Renal Transplantation, Sri Aurobindo Institute of Medical Sciences (SAIMS), \\ Indore (M.P.), India
}

\section{ABSTRACT}

Introduction: Large stones in renal pelvis can be treated with percutaneous nephrolithotomy (PCNL) or pyelolithotomy (either by open or laparoscopic techniques). PCNL is difficult in undilated system. For pyelolithotomy, laparoscopy is more preferable over the open surgery. Surgeons are more familiar with the tansperitoneoscopic anatomy than retroperitoneoscopic one, but retroperitoneoscopic approach can be attempted if we anticipate the problems in the transperitoneal route.

Case: A fifty years old gentleman presented to us with the complaint of dull aching right flank pain. On ultrasonographic examination, he was found to have a large stone in renal pelvis with minimal hydronephrosis and thickened omentum on right side. Xray KUB showed a large radio-opaque shadow in renal area. We did the CECT-Urogram of the patient to know the detailed anatomy, which showed a stone of 5.3 x $3.7 \mathrm{~cm}$ in right extra-rena pelvis without hydronephrosis and a large focal area of marked fat stranding in omentum on the right side in mid and lower abdomen with swirling of fat stranding on the superior aspect suggestive of omental infarction and torsion.

Due to undilated caliceal system, we preferred laparoscopic surgery over the PCNL in this patient. As whole of the omental tissue was stuck on right side we decided to proceed with transperitoneoscopic route instead of retroperitoneoscopic one. The DJ stent was inserted preoperatively.The surgery was performed in the flank position with three ports, one 10mm port just antero-inferior to tip of 12th rib for camera and two $5 \mathrm{~mm}$ working ports, one at anterior axillary line and other at renal angle. We created the retroperitoneal space with the customized balloon, made with the glove-fingure.

Results: The operative time was 1 hour 40 minutes, and there were no intra or post-operative complications. The stone was removed in toto. Patient was orally allowed on first postoperative day and foleys was removed on second day. patient was discharged on day 2.

DJ stent was removed after 15 days. At two months follow-up the patient was asymptomatic.

Conclusion: The retroperitoneoscopic pyelolithotomy is a good alternative for removal of large stone in renal pelvis, with the added advantages of no peritoneal contamination and a quick recovery of bowel function.

\section{ACKNOWLEDGEMENT}

Mr. Aashish and Mr. Himanshu Srivastava from SAIMS, Indore for making animation.

\section{ARTICLE INFO}

Available at: www.brazjurol.com.br/videos/january_february_2014/Chipde_123_124video.htm

Int Braz J Urol. 2014; 40 (Video \#1): 123-4 
Submitted for publication:

December 01, 2013

Accepted after revision:

January 30, 2014
Correspondence address:

Saurabh Sudhir Chipde, MS MCh

Assistant Professor, Department of Urology and Renal Transplantation,

Sri Aurobindo Institute of Medical Sciences (SAIMS), Indore (M.P.), India. Mobile: 91-9977512542; 7389501402

Email: drsaurabh07@rediffmail.com

\section{EDITORIAL COMMENT}

The video submitted by Drs. Chipde and Agrawal nicely depicts a retroperitoneal, laparoscopic solution for removal of a large renal pelvic stone. This patient's case was further complicated by omental infarction and torsion, making a transperitoneal approach very difficult. In 2013 it is rare to find a case of large kidney stones that cannot be treated with percutaneous nephrolithotomy. The authors mention that the lack of hydronephrosis was a factor in their decision to proceed via a laparoscopic approach. These issues can usually be overcome by various retrograde transurethral techniques Cystoscopy with placement of an open-ended ureteral catheter followed by retrograde ureteropylography while the patient is in the prone position can nicely delineate a non-dilated collecting system and can facilitate percutaneous access (1).

There is a small subset of patients that likely can benefit from laparoscopic stone surgery. They are patients with large kidney stones and associated pathology that requires correction, such as a ureteropelvic junction obstruction or ureteral stricture. In patients with relative contraindications to a transperitoneal approach, the retroperitoneal approach is useful.

Hubert Swana, MD Pediatric Urology Nemours Children's Hospital Orlando Orlando, FL, USA E-mail: hswana@nemours.org 